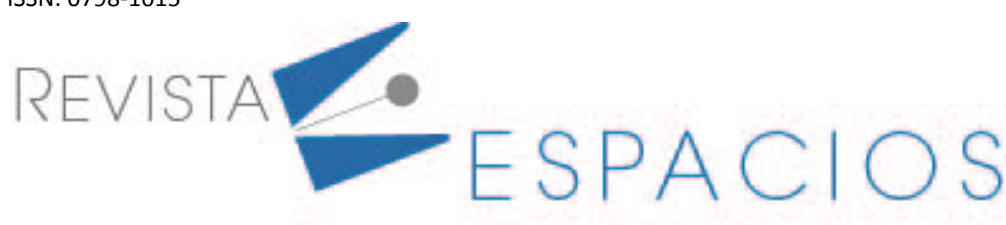

Educación • Education • Educação • Vol. 42 (01) 2021 • Art. 6

\title{
Uso de los videojuegos en básica primaria: una revisión sistemática
}

\section{Use of videogames in primary school: a systematic review}

\author{
MENDEZ, Maribel ${ }^{1}$ \\ BOUDE, Oscar ${ }^{2}$
}

\begin{abstract}
Resumen
Se muestra los resultados de una investigación desarrollada en la Universidad de La Sabana, con el objetivo de identificar los usos que se han dado a los videojuegos en educación primaria en el mundo. Se realizó una revisión sistemática de literatura publicada en revistas científicas entre el 2000 y 2019. Los resultados muestran que el área de mayor uso de videojuegos son las ciencias naturales, y que su uso favorece la interacción y la innovación de las prácticas educativas.

Palabras clave: videojuegos, juegos serios, básica primaria, tecnologías de la información y la comunicación.
\end{abstract}

\begin{abstract}
The results of a research carried out at the University of La Sabana are shown, with the aim of identifying the uses that have been given to video games in primary education in the world. A systematic review of literature published in scientific journals was carried out between 2000 and 2019. The results show that the area of greatest use of video games is the natural sciences, and that their use favors interaction and innovation in educational practices.

key words: videogames, serious games, basic elementary, information and communication technologies.
\end{abstract}

\section{Introducción}

En los últimos años los avances tecnológicos se han incrementado exponencialmente, permitiendo a las personas tener acceso a una diversidad de herramientas y recursos digitales que facilitan la generación de vínculos comunicativos, sociales y culturales (Boude y Sarmiento, 2017). Modificando la forma en que nos comunicamos, relacionamos, aprendemos e interactuamos (Castells, 2004; Mattelart 2007). Lo anterior, ha generado en la educación una diferencia marcada entre las concepciones que tienen los estudiantes y sus docentes sobre cómo se deben integrar las Tecnologías de la Información y Comunicación (TIC) en el aula de clase (Boude y Sarmiento, 2017). Diferencias que dificultan el aprendizaje de los estudiantes, pues generan fricciones dentro del proceso (Boude, 2014). En el mismo sentido, los expertos coinciden en que el proceso de formación tradicional basado en el modelo de transferencia de conocimiento, se ha quedado corto frente a las necesidades actuales de la sociedad (Boude y Sarmiento, 2017). Más aún, todos estos factores exigen cambios en "los contenidos que se

\footnotetext{
${ }^{1}$ Docente-Investigador.Centro de tecnologías para la Academia. Universidad de La Sabana. Maribel.mendez@unisabana.edu.co

${ }^{2}$ Docente-Investigador.Centro de tecnologías para la Academia. Universidad de La Sabana. oscar.boude@unisabana.edu.co
} 
enseñan, las actividades de aprendizaje, la evaluación y las formas de comunicarnos con el alumnado" (Moreira, 2018 p.27).

Todo lo anterior, ha generado en los profesores la necesidad de transformar sus prácticas pedagógicas, involucrando cada vez más una diversidad de recursos tecnológicos (Boude y Sarmiento, 2017), que van desde herramientas Web 2.0 hasta entornos metaversos y videojuegos. No obstante, muchos de estos procesos se hacen como respuesta a las tendencias que en temas de integración de tecnología encuentran en redes sociales y sitios especializados o a través de experiencias exitosas que han sido reportadas por otros docentes en blogs.

Si bien, este proceso no está mal y contribuye a transformar las prácticas, algunas de estas iniciativas no llegan a concretarse pues existen muchas dudas en los docentes alrededor de los diferentes factores que deben tener en cuenta a la hora de integrarlas. Más aún, cuando se trata de integrar videojuegos, pues, aunque es una de las tendencias emergentes en el contexto del Aprendizaje Apoyado en Tecnología (AAP) gracias a su atractivo audiovisual, su carácter desafiante y motivador (Sandoval y Triana, 2017), aún son muchas las interrogantes que tienen los docentes alrededor de cuándo y cómo integrarlos. Debido, a que los videojuegos comerciales están pensados para el entretenimiento y no para apoyar procesos de formación, lo que genera ciertas dificultades para el docente a la hora de integrarlo a las exigencias establecidas en el programa del curso.

Como respuesta a lo anterior, han surgido los Juegos Serios que son videojuegos orientados a construir conocimientos y habilidades desde los contenidos de la escuela, trabajo y salud, su diseño y producción no se limitan solo a entretener sino a promover aprendizajes de manera intencional (Gee, 2009). No obstante, muchos de ellos han fracasado al favorecer la promoción de aprendizajes sobre el entretenimiento. Y es que, el diseño de Juegos serios es un proceso complejo, que implica que el docente se salga de su zona de confort y piense en los diferentes elementos que esperaría encontrar su estudiante dentro del mismo, las mecánicas que les atraen y las dinámicas que necesita favorecer para propiciar las competencias y habilidades que están formulados en el currículo (Felicia, 2009).

Tanto así, que hoy en día, el desarrollo de los videojuegos con fines educativos ha llevado a realizar investigaciones orientadas a encontrar diferentes formas y estrategias de uso para apoyar procesos formativos. Actualmente existen numerosos videojuegos diseñados para el aprendizaje, que se implementan en las aulas de clase y otros espacios de enseñanza, este acelerado desarrollo ha generado interés en diversos campos del saber, por estudiar su pertinencia y efectos en el aprendizaje de diversas habilidades (Starks, 2014).

Por todo lo anterior, este rastreo quiso identificar: los usos que se están dando a los videojuegos en educación básica primaria, las características que poseen aquellos que son exitosos, la relación que existe entre el área disciplinar y el tipo de videojuego más propicio a cada una de ellas, las habilidades y competencias que suelen desarrollar y las tecnologías utilizadas dentro de estos. Con el fin, de brindar a la comunidad académica respuestas a partir de la revisión sistemática de las investigaciones que han sido reportadas desde el año 2000, año en el que inicia a crecer en el número de publicaciones en Scopus alrededor del uso de videojuegos en educación.

Este ejercicio permitió reconocer que, frente a las áreas de aplicación, existe un porcentaje significativo orientado a las ciencias naturales, ciencias sociales y matemáticas. Asimismo, es importante mencionar que, durante la revisión se encontraron otras áreas de uso y textos referidos a otras tecnologías combinadas con videojuegos, los cuales se caracterizaron dentro de las mismas categorías de análisis y se encuentran descritas sus coincidencias en el presente informe. 


\section{Metodología}

Para la realización de esta revisión se utilizó un método de Revisión Integrativa (Fink, 2014), método cuya finalidad es obtención de nuevas conclusiones a partir de los resultados de otras investigaciones ya realizadas. Particularmente para realizar el proceso de revisión de la literatura se siguió el método de Conn y colaboradores (2003). El cual indica que se deben seguir las siguientes etapas: delimitación del objetivo; definición de los criterios de inclusión; búsqueda de los textos en las bases de datos; análisis de los resúmenes; selección de los textos de acuerdo con los criterios de inclusión; evaluación crítica de los mismos y análisis de los datos recolectados.

Para este caso, el propósito primario del estudio fue identificar los usos que se dan a los videojuegos en la enseñanza a nivel de educación básica, así como, las habilidades y competencias que desarrollan, las características, elementos y tecnologías utilizadas dentro de éstos, a partir de las investigaciones que han sido reportadas en esta materia. Ahora bien, con el fin de limitar la revisión a los objetivos declarados y así incluir o excluir adecuadamente los textos durante el proceso, se definieron los siguientes criterios de inclusión:

- Artículos publicados en revistas científicas indexadas en las principales bases de datos.

- Estudios realizados en educación básica primaria que integren videojuegos.

- Estudios disponibles en idiomas castellano, inglés y portugués.

- Estudios publicados entre 2000 y 2019.

Con estos criterios se definió un corpus documental que incluyó artículos publicados en revistas indexadas en las siguientes bases de datos: ISI, Scopus, ProQuest, EBSCOhost, y ScienceDirect, así como, tesis de maestría y doctorado que fueron obtenidas con Google Scholar y repositorios universitarios. Los descriptores de búsqueda utilizados fuero: "Videojuegos + educación primaria, "Juegos serios + básica primaria", "video games + elementary education", "video games + primary Education", "Serious games + primary-elementary ".

Este enfoque produjo un corpus de 160 documentos iniciales luego de revisar los títulos de los textos obtenidos con las palabras claves utilizadas en las diferentes bases de datos. Dicho corpus se redujo a 92 textos luego de comparar e unificar los resultados obtenidos en las diferentes Bases de datos. Para finalizar se realizó un análisis de los resúmenes de los textos antes mencionados y se aplicaron los criterios de inclusión, lo cual limitó el corpus a los 80 textos finales incluidos en la revisión.

Para el proceso de análisis de los datos se siguió el método de análisis temático indicado por Braun y Clarke (2006), el cual consiste en los siguientes procesos: Familiarización de los datos, codificación inicial, búsqueda de patrones, revisión de patrones, definición de patrones y escritura y producción del informe final.

\section{Resultados}

Los datos encontrados fueron analizados y categorizados según las intenciones de la revisión, por tal razón a continuación se indican dichos resultados a la luz de las intenciones indicadas con anterioridad.

\subsection{Usos dados a los videojuegos en Básica primaría}

Los resultados obtenidos dan cuenta de una gran variedad de áreas en las que son utilizados los videojuegos en procesos de formación de básica primaria. Los cuales van desde el apoyo de áreas como Ciencias Naturales, Matemáticas y Ciencias Sociales, hasta su uso en estrategias de inclusión educativa y uso terapéutico de los estudiantes, tal y como se muestra a continuación. 
Figura 1

Distribución de las investigaciones por áreas de implementación

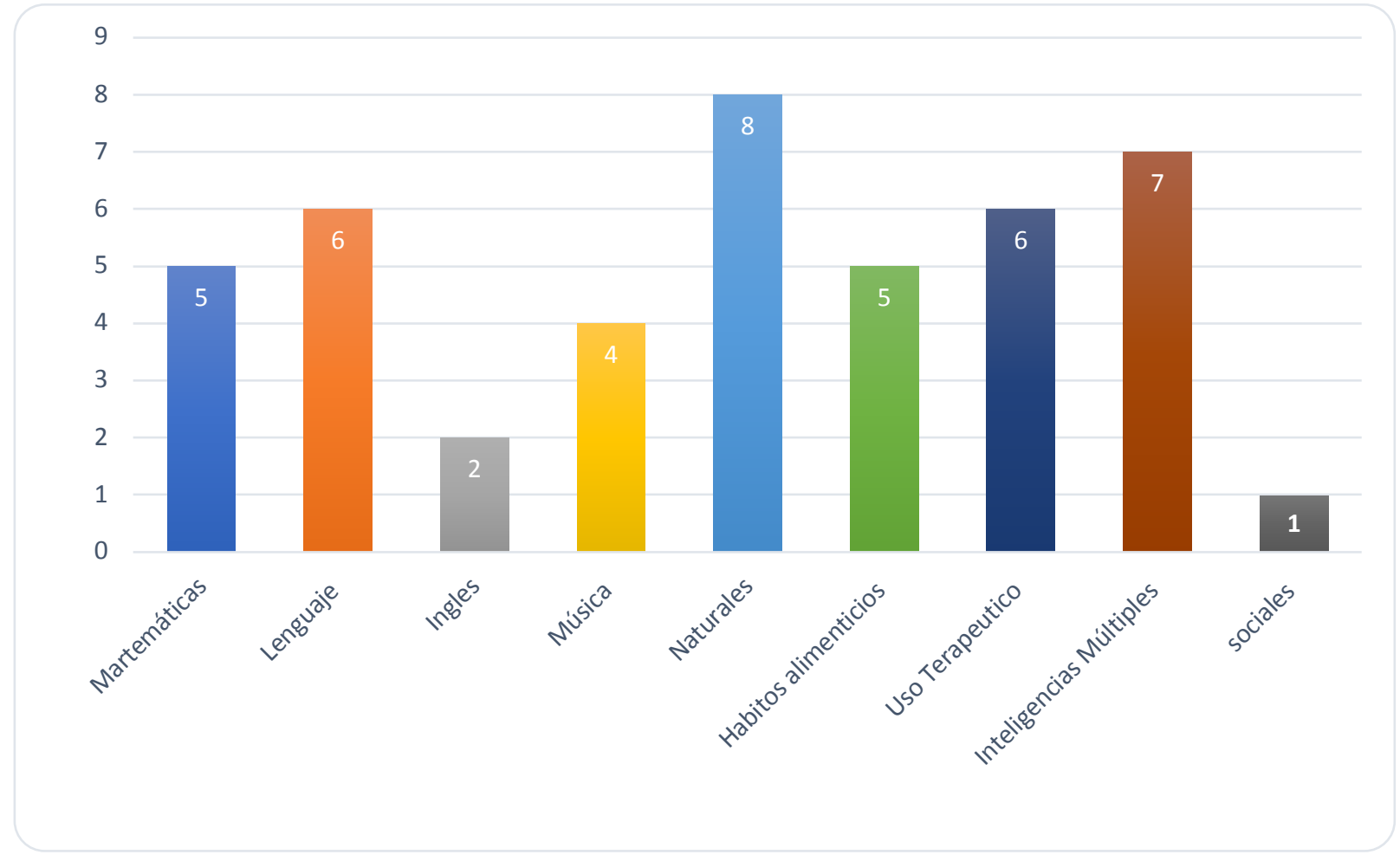

No obstante, al agrupar los resultados se observa que el $46 \%$ los estudios se realizaron en las áreas de ciencias naturales, sociales y matemáticas. De éstos, el $42 \%$ en la enseñanza de matemáticas, $24 \%$ en ciencias sociales y el $24 \%$ restante en ciencias naturales. Ahora bien, a nivel de matemáticas los investigadores reportan que los videojuegos ayudan al desarrollo del cálculo mental, la solución de problemas, la lógica espacial, ubicación e identificación de figuras geométricas y ejercicios prácticos con fraccionarios(Bakker, Van den Heuvel, Robitzsch, 2015; Capell, Tejada y Bosco, 2017; Morales, 2009; Macías y Quintero, 2011; Plass-Nielsen y Nielsen, 2019; Villacís et at., 2015) además, incentiva los niveles de atención gracias a la emoción que les genera su uso (Del Moral y Fernández, 2017; Zhao y Linaza, 2015). Así mismo, los estudiantes están más motivados y con menos temores ante el error, debido a que pueden repetir la actividad o buscar nuevas estrategias para desarrollarla. (Saorin, De la Torre, Melian, Meier y Rivero, 2015).

A nivel de ciencias sociales, los estudios indican que los videojuegos son innovadores y en la medida que se adapten a los currículos de primaria, mejoran los niveles de conocimiento y actitudes de los estudiantes frente al aprendizaje. Además sirven como complemento para las clases de historia, orientación espacial, reconocimiento y ubicación del territorio entre otras. (Bokolas y Panagouli, 2019; Evaristo, et al (2016; Legerén y Doval, (2015); Macías y Quintero, (2011(; Marín y Sampedro, (2016); Sáez y Cózar, (2017).

Por su parte, en Ciencias naturales los investigadores coinciden en que han incrementado el interés, la motivación (Lima, et al, (2015) y la apropiación conceptual de los temas de las ciencias (Lin, et al., (2019); Martínez, Díaz et al., (2013); Solano y Santacruz, (2016), así como, las correlaciones que hacen los estudiantes en los entornos reales (Del Pozo, (2012); Pérez y Pérez, (2015). Asimismo, es importante resaltar que las temáticas más abordadas son la sensibilización ante el cuidado de la fauna procesos de reciclaje, conciencia y cuidado del medio ambiente, niveles de organización de los seres vivos y su reconocimiento en el mundo natural. 
A nivel de otras áreas, contribuyen a desarrollar habilidades para la sociedad del conocimiento, el pensamiento crítico, la alfabetización digital y las nuevas formas de participación (Checa, 2016); Lacasa, et al., (2008) Valderrama, (2012). Así como, el desarrollo de competencias digitales, incentivando la indagación crítica de la información encontrada en la web y su pertinencia. (Berríos, et al, (2015); Chiazzese, et al., (2018)M; Costa, et al (2017); Iten y Petko, (2016); Sáez y Cozar, (2017). El manejo de nuevas tecnologías para el aprendizaje abre ventanas de oportunidad para realizar nuevas lecturas desde lo audiovisual, icónico y gráfico, que dista de la enseñanza tradicional y permite al estudiante tener flexibilidad ante los cambios, propiciando estrategias de innovación y creatividad para desenvolverse en la sociedad (Drew y Bogost, 2005); Marcano, (2010); Solano y Santacruz, 2016). Los videojuegos se pueden considerar como una forma efectiva de alfabetización digital porque se han convertido en una forma de comunicarse, de expresarse e intercambiar conocimientos (Marcano, 2006) además de generar habilidades digitales en los estudiantes y favorecer la adquisición de conceptos (Del Moral, Fernández y Guzmán, (2016); Iten y Petko, (2016); Marín, (2010); Portilla, det al (,2015; Solano y Santacruz,( 2016).

Finalmente, la literatura muestra videojuegos diseñados para el aprendizaje de la música, manejo de pentagramas, conocimiento de instrumentos musicales y de folklore territorial, notándose efectos motivacionales y significativos en el área (Azorín, 2014; García y Raposo, (2013); Ramos y Botella, 2015,) 2016).

\subsection{Usos de videojuegos en áreas fuera del currículo}

Asimismo, los videojuegos han sido utilizados como alternativa terapéutica para mejorar: el desarrollo psicomotor en niños con parálisis cerebral, encontrando efectos positivos en cuanto a control postural y equilibrio (Yagüe, Yagüe, Lekuona y Sanz, 2016), la coordinación y planeación motora en casos de dispraxia y disposición ante las sesiones de terapias (Straker et al., 2015), de atención y tratamiento para mejorar dificultades de lateralidad (Granados y Moreno, 2012), la intervención pedagógica en el tratamiento de la dislexia y conciencia fonológica (Jiménez y Rojas, 2008); Rojas, (2008); Gaggi et al.,( 2017) y la empatía, el conflicto escolar, y el manejo de emociones (Brenes y Pérez, 2015); Filella, et al., 2016). Así como, para la adopción de conductas saludables como el lavado de manos (Kang y Chang, 2019) y lograr que chicos con autismo tomen una ducha de forma independiente (Kang y Chang, 2019).

La creación y uso de videojuegos para promover hábitos alimenticios como tratamiento y prevención de la obesidad infantil, cuyas investigaciones coinciden que, su uso incentivó la adopción de conductas saludables de alimentación, proporcionando motivación y entretenimiento al experimentar y valorar diferentes situaciones de juego al tomar decisiones acertadas respecto a la alimentación para mejorar la salud ( Villaseñor, et al.,( 2013; López et al., 2013; Druzhinenkoa, et al., (2014). Otros por su parte hallaron en los videojuegos una excusa para desarrollar conciencia frente a la hambruna infantil, creando estrategias para llevar ayudas humanitarias a otros territorios (Petros, et al (2011).

\subsection{Habilidades y competencias desarrolladas con el uso de videojuegos en las investigaciones}

Debido a que los videojuegos le dan al jugador un ambiente libre de sesgos (Geymonat, 2014); Tejada y Bosco; (2017) en el que puede experimentar y resolver un conjunto de problemas con creatividad y motivación (Valderrama, 2012); Domínguez y Saéz, (2014) a través, de procesos reflexivos; el jugador logra desarrollar habilidades que le permiten organizar la información para solucionar problemas, mejorar la planificación al desarrollar cálculos mentales, gestionar recursos y tomar decisiones (Gros, 2000); Morales (2009); Obikwelu, Read y Sim, (2012); Capell, et al., (2017). 
Los estudios resaltan que el uso de videojuegos aumenta la creatividad, y promueve el desarrollo de prácticas creativas de enseñanza (Frossard, 2013; Geymonat, 2014). Gracias a que estos permiten al docente transformar sus prácticas docentes (Torrente et al., 2009), diseñar diferentes estrategias para su uso dentro y fuera de clase (Salvador et al., 2012); Beavis, Muspratt y Thompson, (2014) y mejorar la capacidad de los alumnos para aplicar los conocimientos a la vida real (Del Moral et al, 2016).

Ahora bien, la lectura de los lenguajes icónicos y gráficos dentro del videojuego implica la comprensión de códigos lingüísticos que permiten llevar a la comprensión de diferentes temáticas. Varias investigaciones abordaron el desarrollo de habilidades comunicativas en las cuales el objetivo era acompañar los procesos de lectura y escritura, ampliar el vocabulario o aprender otro idioma (Moreno y Barriga, (2011); Maniega, Yanèz y Lara, (2011); Fisser, et al., (2013); Gómez, et al.,( (2015); Del Pozo, 2013).

\subsection{Características de los videojuegos usados para la enseñanza}

Los videojuegos exitosos en educación suelen incluir elementos de estimulación sensorial como movimientos y sonidos ensamblados en el contenido gráfico, la interacción personaje para lograr la superación de retos y el diseño en tercera persona para apreciar las acciones de los personajes en cada escenario, proporcionando diversión y entretenimiento a los estudiantes (Marcano,2006); Anyaegbu, Ting, Li, (2012); Santamaría y Mendoza, (2012); Lima et al., (2015); Ramos y Botella, (2016). Y es esto último, lo que lleva a los estudiantes a interactuar con la tecnología y lograr un mayor interés por sus aprendizajes Sim et al., (2015); Rojas (2008). Más aún, Marín (2010) indica que integrar temáticas culturales y sociales en la interfaz del videojuego permite: acercar a los estudiantes al conocimiento de manera atractiva e interactiva, al reconocer situaciones de su contexto, posibilita el aprendizaje activo y experiencial, así como, asociar y correlacionar aprendizajes y tal y como ha socedido en ámbitos industriales y militares (Sandoval y Triana, 2017).

Ahora bien, la interacción logra involucrar emocionalmente al jugador, ya que le permite convertirse en el protagonista de la historia que va construyendo (Portillo, Rodríguez y Fuentes, 2015; Mejía, 2012). A tal punto, que cuando el estudiante logra engancharse al mundo virtual, el videojuego se convierte en un centro de solución de situaciones o entornos reales en donde el estudiante demuestra sus conocimientos (Contreras, et al.,(2011; Maniega, et al., (2011); Zhao y Linaza, (2015); Díaz et al., (2015).

No obstante, es fundamental articular la integración de los videojuegos a los contenidos curriculares del curso, y para esto las estrategias más usuales suele ser, utilizarlos como complemento de la práctica docente, apoyo a los contenidos de libros de texto y como herramientas didácticas para integrar temáticas transversales (Del Pozo, 2013); Solano y Santa Cruz, (2016); Androussou, Kourti y Askooni, (2013). Además, Sánchez, Ruiz y Sánchez (2017) confirman que, si los videojuegos están bien adaptados al currículo estos logran tener una incidencia positiva en los aprendizajes de los estudiantes, en contraste, con aquellos que reciben clases tradicionales.

Para finalizar, los videojuegos tipo multijugador suelen incrementar la interacción (Gros,2000), fomentar la participación y motivación porque los jugadores pueden explorar mundos de manera alterna con otros jugadores, lo que hace que haya mayor y mejor inmersión (Zhao y Linaza, 2015); Capell, Tejada y Bosco, (2017). Lo anterior, propicia espacios colaborativos en los cuales los jugadores intercambian información para lograr las metas del juego y por ende metas en los aprendizajes (Androussou, Kuorti y Askouni, (2013). Asimismo, (Yagüe et al., 2015) postulan que este tipo de juegos permiten la integración social favoreciendo el intercambio de saberes. A continuación se muestra las diferentes características identificadas. 
Tabla 1

Características de los videojuegos usados en primaria

\begin{tabular}{|c|c|c|}
\hline $\mathrm{N}^{\circ}$ & $\begin{array}{l}\text { Características más relevantes de los } \\
\text { videojuegos }\end{array}$ & Referencias \\
\hline 1 & $\begin{array}{l}\text { Interacción de los elementos de videojuegos } \\
\text { como factor de motivación para el aprendizaje }\end{array}$ & $\begin{array}{l}\text { (Marcano, 2006; Rojas, 2008; Mejía, 2012; Lima et al., 2015; } \\
\text { Sim, Read, Gregory y Xu, 2015; Portilla, Rodríguez y Fuentes, } \\
\text { 2015; Ramos y Botella, 2016) }\end{array}$ \\
\hline 2 & $\begin{array}{l}\text { Componentes inmersivos y su efecto en el } \\
\text { aprendizaje }\end{array}$ & $\begin{array}{l}\text { (Contreras, Eguía y Solano, 2011; Maniega, Yánez, y Lara, 2011; } \\
\text { Valderrama, 2012; Díaz et al., 2015; Zhao y Linaza, 2015) }\end{array}$ \\
\hline 3 & Elementos de gratificación emocional & (Marcano, 2006) \\
\hline 4 & Adaptación de temáticas curriculares & $\begin{array}{l}\text { (Torrente, Moreno, Martínez y Fernández, 2009; Martín del } \\
\text { Pozo, 2013; Androussou, Kourti y Askooni,2013; Marín Y } \\
\text { Martín, 2014; Ramos y Botella, 2016; Solano y Santacruz, 2016; } \\
\text { Iten y Petko, 2016) }\end{array}$ \\
\hline 5 & $\begin{array}{l}\text { Diseño del videojuego relacionado con } \\
\text { entornos reales }\end{array}$ & $\begin{array}{l}\text { (Marín, 2010; Martín del Pozo, 2013; Androussou et al., 2013; } \\
\text { Villaseñor et al., 2013) }\end{array}$ \\
\hline 6 & Presentar simuladores & $\begin{array}{l}\text { (Valderrama, 2012; Starks, 2014; Tellez, 2015; Sandoval y } \\
\text { Triana, 2017) }\end{array}$ \\
\hline 7 & Presentar posibilidades de juego multijugador & $\begin{array}{l}\text { (Gros, 2000; Androussou et al., 2013; Martín del Pozo, 2013; } \\
\text { Zhao y Linaza, 2015; Capell et al., 2017) }\end{array}$ \\
\hline 8 & Fácil navegación & (Martínez etal., 2013) \\
\hline 9 & Obtener feedback y puntuaciones & $\begin{array}{l}\text { (Obikwelu, Read y Sim, 2012; Martínez, Díaz et al., 2013; Eguía, } \\
\text { Contreras, Solano, 2014; Sim, Read, Gregory y Xu, 2015; Iten y } \\
\text { Petko, 2016) }\end{array}$ \\
\hline
\end{tabular}

\subsection{Tipos de videojuegos utilizados}

Aunque no todas las investigaciones indican el tipo de videojuego utilizado, fue posible determinar que la mayoría de estudios utilizaron los de aventura gráfica, ya que invitan al jugador a interactuar con el escenario y los personajes, también a socializar con otros jugadores para comprender las formas que se pueden superar las metas de juego (Mejía, 2012). En segundo lugar, están los tipos arcade que, por su manejo intuitivo, favorece el desarrollo motor, direccionalidad y manejo del espacio, así como también conocimiento en reglas y estereotipos (Marín, 2010). En tercer lugar están los juegos de simulación, los cuales brindan experiencias desde entornos y situaciones reales recreadas en el mundo virtual. En menor medida se encuentran los de rol, en los cuales el jugador se identifica con un personaje dentro del juego, asumiendo actitudes, valores, prácticas y habilidades que son proyectadas en contextos reales.

\subsection{Otras tecnologías integradas con videojuegos para apoyar proceso de aprendizaje}

La revisión permitió detectar que junto a los videojuegos, los investigadores han utilizado otras tecnologías como la Realidad Aumentada (RA), ya que, su uso permite mejorar la capacidad para realizar las tareas de juego, potenciar la memoria visuoespacial, detectar y prevenir problemas de aprendizaje futuros y principalmente aumento de la motivación, al estar ante un ambiente diferente de aprendizaje (Martínez, et al., 2013; Lin, et al., 2016).

En temáticas relacionadas a las matemáticas y las ciencias, el aprendizaje de la geometría, clasificación de elementos, uso del tangram, clasificación de seres vivos, diseño de zoológicos y vida salvaje, son los que más se destacan hasta el momento (Carrillo y Montalvo, 2016; Martínez, et al., 2013; Solano, Casas y Guevara, 2015; Lin, et al., 2016).Por su parte, la realidad virtual (RV) además de generar total inmersión al jugador, contribuye a superar superar dificultades de lateralidad y fortalecer el pensamiento espacial a partir de modelado de elementos geométricos, respectivamente (Santamaria y Mendoza, 2011; Mejía, 2012). También se suele utilizar 
la impresión de modelados en 3D para la apropiación de conceptos geométricos y contrastar las dimensiones digitales de los objetos, contra las físicas (Saorin et al, 2015).

En el mismo sentido, el Kinect es un dispositivo que utiliza monitores de movimiento en una cámara siguiendo los movimientos del cuerpo completo (Straker et al, 2015), ha sido un accesorio utilizado en investigaciones como complemento a terapia física de niños que presentan dispraxia, el uso del Kinect favorece la planeación y organización de los movimientos, que mejoran los procesos psicomotrices en los jugadores. De igual manera se ha usado para promover los buenos hábitos alimenticios y combatir la obesidad infantil, el uso del Kinect motiva a los jugadores a realizar movimientos, mientras avanza en los niveles del videojuego (López, Miranda y González, 2013).

De igual manera, se articula al uso de videojuegos, la robótica educativa; en su investigación, Díaz y colaboradores (2015), enfatizan el aprendizaje de la programación a través de un videojuego, en el cual los niños deben lograr que el robot haga movimientos. Este ejercicio implica coordinación, cooperación y creatividad, por lo cual los investigadores concluyen que, estas prácticas pueden llegar a disminuir la brecha digital existente, proporcionando en las dinámicas educativas nuevos y mejores ambientes cercanos a los estudiantes, que motiven y logren aprendizajes significativos que conlleven al desarrollo del pensamiento crítico y solución de problemas en la sociedad actual.

\section{Conclusiones}

Una de los primeros aspectos que llamo la atención durante el proceso de análisis de la revisión, es que el uso de los videojuegos en primaria no está limitado al desarrollo de las competencias en áreas como la matemática y las ciencias. Sino que, por el contrario, están siendo utilizados en casi todas las áreas del currículo, lo que implica un cambio de postura sobre los videojuegos por parte de las instituciones y los docentes, quienes ya no los ven como un agente distractor dentro del proceso de aprendizaje (Felicia, 2009).

En el mismo sentido, es importante resaltar que además de fortalecer el proceso de formación, los videojuegos están siendo utilizados para fortalecer a los niños en aspectos relacionados con dificultades de aprendizaje, necesidades educativas especiales y modificación de conductas (Gaggi et al., 2017). Los cuales, tienen una incidencia directa en la motivación, disposición y el rendimiento académico de los niños durante su proceso escolar.

Ahora bien, las investigaciones orientadas desde el área matemática reportan que los videojuegos logran desarrollar capacidades analíticas, potenciando los procesos de lógica y entrenamiento de solución de problemas. Por su parte, las investigaciones orientadas desde las ciencias sociales facilitan los aprendizajes de historia, orientación espacial y procesos sociales cooperativos por la motivación que genera, y a nivel de ciencias naturales, las investigaciones indican que facilitan la sensibilización frente al cuidado del medio ambiente y el reconocimiento de seres vivos, estos estudios coinciden en que la implementación de videojuegos en el área permite crear conciencia crítica que invita al cuidado del entorno de manera activa.

Pero, independientemente del área o uso que se le dé a los videojuegos, todos los estudios coinciden en la importancia que tiene el nivel de interactividad que posea el videojuego para lograr establecer dinámicas participativas.

Por otro lado, temáticas como las habilidades comunicativas, procesos de lectura y escritura, aprendizajes de idiomas y música, forman otro grupo de textos en los cuales se resalta, las posibilidades que brindan los videojuegos, para transformar las prácticas docentes de los profesores, ofreciéndoles una diversidad de mecánicas y dinámicas que hacen que el proceso de formación sea más divertido y atractivo para los estudiantes. 
No obstante, es importante mencionar que como cualquier otra tecnología el uso prolongado de la misma hará que se pierda el efecto novedad y disminuya la motivación, convirtiendo este proceso en una actividad cotidiana de la cual se cansen los estudiantes.

Asimismo, es importante mencionar que, al pensar la integración de videojuegos a los currículos educativos y las prácticas de enseñanza, los profesores tienen un rol fundamental en cuanto a los objetivos e intención pedagógica del uso de videojuegos en las aulas o fuera de éstas, puesto que para que haya un verdadero efecto en la enseñanza y el aprendizaje se necesita promover el uso de la herramienta como una estrategia práctica de aplicación de los conocimientos.

Para finalizar, aunque la revisión muestra un cambio en la percepción que se tiene del uso de videojuegos en educación, aún son muy pocos los estudios reportados al respecto y quedan muchas interrogantes sobre la ventajas y desventajas que tiene incorporar este tipo de tecnología en el proceso educativo, así como el desarrollo de estudios longitudinales sobre los riesgos y beneficios que tiene su uso a largo plazo.

\section{Referencias bibliográficas}

Androussou, A., Kourti, E. y Askouni, N. (2013). "The chest that longs to be moved": A serious game for the greek muslim minority children. European Conference on Games Based learning. 27-34.

Anyaegbu, R; Ting, W. y Li, Y (January 2012). Serius game motivation in an EFL classroom in chinese primary school. TOJET: The Turkish Oneline Jurnalof Educational Technology. 11(1) 154-164.

Area Moreira, M. (2018). Hacia la universidad digital: ¿dónde estamos y a dónde vamos? RIED. Revista Iberoamericana de Educación a Distancia, 21(2), pp. 25-30. doi: http://dx.doi.org/10.5944/ried.21.2.21801

Azorín Delegido, J.M. (2014). El videojuego musical ¿Un recurso para la educación musical en primaria? Ensayos, Revista de la facultad de educación de Albacete 29(2). 19-36.

Bakker, M., Van den Heuvel Panhuizen y M., Robirtzch, A. (2015). Effects of playing mathematics computer games on primary school students multiplicative reasoning ability. British Journal of Educational Technology. 47(4). 633-648.

Beavis, C., Muspratt, S. y Thompson. (2014). Computer games can get your brain working: student experience and perceptions of digital games in the classroom. Journal Learning, Media and Technology. (40). 21-42. DOI: https://doi.org/10.1080/17439884.2014.904339

Berríos-Valenzuela, L., Buxarrais-Estrada, M. R. y Garces, M. S. (2015). ICT use and parental mediation perceived by chilean children. Comunicar, 23(45). 161-168.

Bokolas, V. y Panagouli, D. (2019). Between "fortnite" and "civilization": Digital games and historical-cultural education. Paper presented at the Proceedings of the European Conference on Games-Based Learning, , 2019-October 74-81. doi:10.34190/GBL.19.160

Boude, O. (2014). Caracterización de los usos de los dispositivos móviles en el proceso de formación. Presentado en XV Encuentro internacional Virtual Educa Perú.

Boude, O., y Sarmiento, A. (2017). El reto de formar a profesores universitarios para integrar el aprendizaje móvil. Educación Médica Superior, 31(1), 61-77. 
Brenes Peralta, C., Pérez Sánchez R. (2015). Empatía y agresión en el uso de video juegos en niños y niñas. Revista Latinoamericana en Ciencias Sociales, Niñez y Juventud. Universidad de Manizales, Colombia. (13) 183-194.

Capell Masip, N., y Tejada Fernández, J., y Bosco, A. (2017). Los videojuegos como medio de aprendizaje: un estudio de caso en matemáticas en educación primaria. Pixil-Bit. Revista de Medios y Educación, (51), 133150.

Castells, M. (2004). La era de la información: economía, sociedad y cultura (Vol. 3). siglo XXI.

Conn, V.S., Isamaramalai, S., Rath, S., Jantarakupt, P., Wadhawan, R. y Dash, Y. (2013). Beyond MEDLINE for Literature Searches. Jurnal of Nursing Scholarship, 35(2), 177-182. DOI: http://doi.org/ccpweg).

Contreras, R.S y Eguía, J.L. (2012). Videojuegos en dispositivos móviles para desarrollar competencias en alumnos de primaria. Mobile Comunication 2012, Experiencies i recerques sobre comunicación móbi. Universitat politécnica de Catalunya. 56-64.

Costa, C., Tyner, K., Henriques, S. y Suosa, C. (2017). Digital Game Creation for Media and Information Literacy Development in Children. Academic Conferences \& Publishing. 122-121.

Checa, R.M. (2016). Developing skills in digical contexts: Video game and films aslearning tools at primary school. Games and Culture. Departamento f Education, University of Alcalá, Aulario María de Guzmán. Madrid- España. 11(5). 463-488.

Chiazzese, G., Fulantelli, G., Pipitone, V. y Taibi, D. (2018). Engaging Promary School Chindren in Computational Thinking: Designing and developing videogames. EKS, 19(2). 63-81.

Del Moral Pérez, M., Fernández Garcia, L y Guzmán Duque, A. (2016). Proyecto game to learn: aprendizaje basado en juegos para potenciar las inteligencias lógico matemáticas, naturalista y lingüística en educación primaria. Píxel-Bit. Revista de Medios y educación, (49), 173-193.

Del Moral Pérez, M y Fernández García, L. C. (2017). Videojuegos en las aulas: implicaciones de una innovación disruptiva para desarrollar las inteligencias Múltiples. Revista Complutense de Educación. (26), 97-118.

Del Pozo, M. (2012). Videojuegos y Educación Ambiental en Educación Primaria. III Congreso Internacional comunicación 3.0, Las Media Enterprises y las Industrias Culturales, Investigara la Comunicación y los nuevos medios. Universidad de Salamanca. 2-19.

Del Pozo, M. (2013) Creación de un videojuego educativo para trabajar los colores y la lengua inglesa en educación primaria. Congreso Internacional de videojuegos y educación, Universidad de Salamanca. 15817.

Díaz, J., Queiruga, C., Tzancoff, C. B., Fava, L., y Harari, V. (2015). Robótica Educativa y Videojuegos en el Aula de la escuela. Laboratorio de Investigacion en Nuevas Tecnologías Informáticas (LINTI). Facultad de informática Universidad nacional de la Plata , 2-9. La Plata, Argentina.

Domínguez Garrido, C. y Saéz López, J.M. (2014). Integración pedagógica de la aplicación Minecraf Edu en esducación primaria: Un estudio de caso. Revista de Medios y Educación, Universidad de Sevilla. (45). 95110.

Drew, D., Bogost, I. Videogames and the future for education. On The Horizon. 13(1). 119-125. 
Druzhinenkoa, D.A., Podolskyb, A.I., Podolskiyc, O.A y Schmolla. (2014). Using Videogames to Treat childhood Obesity. Psychology in Russia: State of the Art. 7(4).

Eguía Gómez, J.L y Contreras Espinosa, R.S (2011). El videojuego como recurso para el aprendizaje estratégico en las aulas: el caso de personatges in Joc. (Tesis de grado doctorado) Universitat de Vic, Departament Comunicació. 258. http://hdl.handle.net/10803/124835

Eguía Gómez, J.L., Contreras Espinosa, R.S., Albajes Solano Lluis. (2014) Co- creación de un juego serio para desarrollar competencias en el currículo de educación primaria. Conferencia Ibérica de Sistemas tecnología de la Información. (1). 993-998.

Evaristo Chiyong, I.S., Vega Velarde, M.V., Navarro Fernandez, R.y Nakano Osorio, T. (2016). Uso de un videojuego educativo como herramienta para aprender historia del Perú. Revista Iberoamericana de educación a Distancia RED. 19(2). DOI: https://doi.org/10.5944/ried.19.2.15569

Felicia, P. (2009). Videojuegos en el aula. Manual para docentes. European schoolnet.

Fisser, P., Voogt, J. y Bom, M. (2013). Word Score: A serious vocabulary game for primary school underachievers. Education and information technologies. 18(2) 175-178.

Filella, G., Cabello, E., Pérez, N y Ros. (2016). Evaluation of the Emotional Education program "Happy 8-12" for the assertive resolution of conflicts among peers. Electronic Journal of Research. 14 (3). 582-601. http://dx.doi.org/10.14204/ejrep.40.15164

Fink, A. (2014). Conducting research literature reviews: From the internet to paper. Sage publications.

Frossard, F. (2013). Fostering teachers creativity through the creation of GBL scenarios. Tesis Doctoral. Universidad de Barcelona. España. DOI: edsair.dedup.wf. 001..3454c84f62f490f5088e578269f616bf.

Gaggi, O., Palazzi, C.E., Ciman, M., Galiazzo, G., Franceschini, S., Ruffino, M., Gori, S. y Facoetti, A. (2017). Serious games for early identification of developmental Dyslexia. Computers in Entertaiment. 15 (2). p12424.

García Rodríguez, F., y Raposo Rivas, M. (2013). Trabajando con videojuegos en el aula una experiencia con wii music. Tendencias Pedagógicas (22) 45-58. https://revistas.uam.es/tendenciaspedagogicas/article/view/2041/2149

Gee, J. P. (2003). What videogames have to teach us teach us about. Learning and Literacy. New York. Palagrave. Micmillan, 2 (14) 203-210.

Gee, J.P. (2009). Theories and mechanisms seriousgame for learning chapter 5: Deep learning properties of Good digital games, Howfar can they (60)

Granados Santamaría, L. y Moreno Mendoza, J.F. (2012). Escenarios virtuales para apoyar el desarrollo de destrezas en niños con dificultades de lateralidad. Revista de educación y desarrollo Social. 6(1). 119-133.

Iten, $\mathrm{N}$ y Petko, D. (2016). Learning with serious games: Is fun playing the game a predictor of learning success?. British Journal of Educational Technology. 47(1). 151-163 DOI: 10.1111/bjet.12226

Jiménez, J.E. y Rojas Martínez E. (2008). Efectos del videojuego tradislexia en la conciencia fonológica y reconocimiento de palabras en niños disléxicos. Psicothema, 20 (3), 347-353. 
Kang, Y y Chang, Y. (2019). Using a motion-controlled game to teach four elementary school children with intellectual disabilities to improve hand hygiene. Journal of Applied Research in Intellectual Disabilities, 32(4), 942-951. doi:10.1111/jar.12587

Kang, Y y Chang, Y. (2019). Using game technology to teach six elementary school children with autism to take a shower independently. Developmental Neurorehabilitation, 22(5), 329-337.

doi:10.1080/17518423.2018.1501778

Lacasa, P., Martínez, R y Méndez, L. (2008). Developing new literacies using comercial videogames as Educational tools. In Linguistics and Education.19(2). 85-106.

Legerén, B y Doval, M.I. (2015) We are european citizens. Jugar para aprender a ser europeos. Education in the Knowledge Society, Universidad de Salamanca. 2 (16)49-68.

Lin, C.Y., Chai, H.C., Wang, J.Y., Chen, J., Liu, Y.H., Chen, C.W y Huang, Y.H. (2016). Augmented reality in Educational activities for children whith desabiloties. Journal Elsevier B.V. DOI: DOI:

10.1016/j.displa.2015.02.004

Lin, Y. , Hsieh, Y., Hou, H. y Wang, S. (2019). Exploring students' learning and gaming performance as well as attention through a drill-based gaming experience for environmental education. Journal of Computers in Education, 6(3), 315-334. doi:10.1007/s40692-019-00130-y

Lima, L., Torres, D y Ramírez, E. (2015) Un juego serio para la preservación de la fauna silvestre en peligro de extinción en Venezuela. Tercera conferencia Nacional de Computación, informática y sistemas/ CoNClsa 2015/. Universidad de Carabobo, Valencia, Venezuela. 50-59.

López, M J., Miranda, P. C. y González, S. (2013). Una metodología para el análisis y diseño de un videojuego serio para combatir la obesidad infantil en el estado de Yucatán. Revista iberoamericana para la investigación y el desarrollo educativo 2 (10).

Maniega, D., Yánez, P y Lara, P. (2011). Uso de un videojuego inmersivo 3D para el aprendizaje del español: El caso de "Lost in La Mancha". Revista Icono 14. 9(2). 87-96.

Marín Díaz, V. (2010). Trabajar la educación primaria e inclusiva desde los videojuegos. Revista de Tecnología de la información y comunicación en educación. Facultad de Ciencias de la educación. universidad de Córdoba España. 4(2).

Marín Díaz, V y Martin, P. (2014). Can videogames be used to develop the infant stage Educational curriculum. Journal of New Approaches in Educational Research. 3 (1). 20-25.

Marín Díaz, V. y Sampedro Requena, B.E. (2016). Innovando en el aula de educación primaria con GT6. Innoeduca International Journal of Technology and Educational Innovation. 2(1). 13-19. DOI: http://dx.doi.org/10.20548/innoeduca.2016.v2i1.1061

Marcano, B. E. (2006). Estimulación emocional de los videojuegos: efectos en el aprendizaje. Revista Electrónica Teoría de la educación. Educación y Cultura en la Sociedad de la información. Universidad de Salamanca España, 7(2), 128-140

Marcano, B.E. (2010). Competencias digitales y videojuegos online. Didáctica, Innovación y Multimedia. (19). DOI: https://www.raco.cat/index.php/DIM/article/view/214709/285001

Macías Gutiérrez, G. y Quintero Zazueta, R. (2011). Los videojuegos como una alternativa para el estudio y desarrollo de la orientación espacial. Investigación en Educación Matemática XV. 405-416. 
Mattelart, A. (2007), Historia de la sociedad de la información, Paidós, Barcelona

Martínez, M., Díaz Pernas, J. L., Barroso Martinez, L., Ortega, D y Anton Rodriguez, M. (2013) Movile Serious Game Using Augmented Reality for Supporting Children's Learning About Animals. Procedia Computer Science. (25), 375-381) https://doi.org/10.1016/j.procs.2013.11.046

Mejía Franco, D. (2012). Elementos estructurales de los videojuegos potencialmente educativos para el desarrollo de temáticas escolares relacionadas con el pensamiento espacial en niños y niñas entre ocho y diez años. Tesis Doctoral, Universidad de Manizales, Colombia.

Morales, E. (2009). Uso de los videojuegos como recurso de aprendizaje en educación primaria y teoría de la comunicación. Revista académica de la Federación Latinoamericana de facultades de comunicación social. Universidad Complutence de Madrid.

Moreno Moreno, W.J., y Barriga Velázquez, D. J. (2011). Los videojuegos como mediación pedagógica para la enseñanza de la lengua castellana y potenciar habilidades comunicativas en los estudiantes de grado quinto del colegio José Francisco Socarrás. (Tesis de pregrado) Licenciatura en Lengua Castellana. Repositorio Universidad Javeriana. Bogotá, Colombia.

Obikwelu, C., Read, J y Sim, G. (2012). The Serious Game Approach to Problem-Based Learning for the Dependent Learner. Proceedings of the European Conference on Games Based Learning. 1(2). 356-363.

Padilla Zea, N., y Collazos Ordonez, C., Gutiérrez Vela, F., y Medina, N. (2012). Videojuegos educativos: Teorias y rpopuestas para el aprendizaje en grupo. Ciencia e ingeniería Neograndina, 22(1), 139-150.

Plass-Nielsen, J., y Nielsen, O. B. W. (2019). How to enhance interest in mathematics by using game-based learning. Paper presented at the Proceedings of the European Conference on Games-Based Learning, , 2019-October 1024-1027. doi:10.34190/GBL.19.073

Pérez, J y Pérez, J. (2015) Efecto del videojuego "Blue Sky" para el aprendizaje del manejo de residuos sólidos en niños del nivel primario. Aportes Universitarios. Universidad Peruana Unión. 5(1). 163-172.

Petros, P., Georgios, F y Fesakis. (2011). Educational Aplications of Serious Games: The Case of the Game food Force in Primary Education Students. Academic Conferences \& Publishing International Ltd. 476-485.

Portillo, Y., Rodriguez, R. y Fuentes, A. (2015). Estudio de los videojuegos en Cuba. Gemany, urope: Unpublished. https://doi-org.ez.unisabana.edu.co/10.13140/RG.2.1.3338.9047

Ramos Ahijado, S., y Botella Nicolás, A. (2016). La integración del videojuego educativo con el floklore. Una propuesta de educación en educación primaria. Revista Electrónica Interuniversitaria de formación del profesorado ( Continuacion de la antigua revista de escuealas normales), 19(3), 115-121. doi: http://dx.doi.org/10.6018/reifop.19.3.267281

Ramos Ahijado, S., y Botella Nicolás, A. (2015). Videojuegos y musicomovigramas. Innovación y recursos para el aprendizaje en educación primaria. Opción. 31(1).

Rojas Rodriguez, E. (2008). Diseño y Validación de un Videojuego para el tratamiento de la dislexia. (Tesis de grado doctorado) Universidad de la Laguna, España.

Sáez López, J.M. y Cózar Gutiérrez, R. (2017). Programación visual por bloques en educación primaria: Aprendiendo y creando contenidos en ciencias sociales. Revista Complutense de Educación. Universidad Complutense de Madrid, España. 28(2). 409-426. 
Salvador, G., Pérez, D., Ortega, M., Soto, E., Alcañiz, M y Contero, M. (2012) Evaluación o fan Augmented Reality Enhanced Tabletop System as a Collaborative Learning Tool: A Case Study on Mathematics at the primary School. The Eurographics Asssociation.

DOI:http://dx.doi.org/10.2312/conf/EG2012/education/009-016.

Sánchez, E., Ruiz, J y Sanchez, J. (2017). Videojuegos frente a fichas impresas en la intervención didáctica con alumnos con necesidades educativas especiales. Educar, Universitat Autónoma de Barcelona. (3) 1. 29-48.

Sandoval Forero, C.G y Triana Sánchez, A. (2017). El videojuego como herramienta prosocial: implicaciones y aplicaciones para la reconstrucción en Colombia. Instituto de estudios Políticos y Relaciones Internacionales. Universidad Nacional de Colombia - Sede Bogotá.

Santamaría, G y Mendoza, J.F. (2012). Escenarios virtuales para apoyar el desarrollo de destrezas en niños con dificultades de lateralidad. Educación y desarrollo social. 6(1). 119-133.

Saorin, J.L., De la Torre, C., Melian, D., Meier, C y Rivero, D. (2015) Blokify: Juego de modelado e impresión 3D en tableta digital para el aprendizaje de vistas normalizadas y perspectiva. Digital Education Review (27) 105-121. DOI: http://greav.ub.edu/der/

Sim, G., Read, J.C., Gregory, P. y Xu, D. (2014) From England to Uganda: Children Designing and Evaluating Serious Games. Human- Computer Interaction,(30) 263-293 https://doi.org/10.1080/07370024.2014.984034

Solano Villanueva, C.A., Casas Díaz, J:F y Guevara Bolaños, J.C. (2015). Aplicación móvil de realidad aumentada para la enseñanza de la clasificación de los seres vivos a niños de tercer grado. Revista Ingeniería, Universidad Distrital, Bogotá, Colombia (20) 1.

Solano, N. L., y Santacruz, V. L. (2016). Videojuegos como herramienta en Educación Primaria: caso de estudios con eAdventure. TE\&ET Revista Iberoamericanade Tecnología en Educación y Educación en Tecnología(18), 101-112. DOI: http://teyet-revista.info.unlp.edu.ar/TEyET/article/view/375

Starks, K. (2014) Cognitive behavioral game desing: a unified model for desing serious game. Hypothesis and theory Article. https://doi.org/10.3389/fpsyg.2014.00028

Straker, L., Howie, E., Smith, A., Jensen, L., Piek, J. y Cambell, A. (2015) A crossover randomised and controlled trial of the impacto $f$ active video game on motor coordination and perception of physical ability in chindren at risk of developmental coordination disorder. Human Movement Science. Physiotherapy and Exercise Science, Curtin University, Australia. (42),146- 160.

Torrente, J., Moreno-Ger, P. y Martínez-Ortiz, I., \& Fernandez-Manjon, B. (2009). Integration and Deployment of Educational Games in e-Learning Environments: The Learning Object Model Meets Educational Gaming. Educational Technology \& Society, 12 (4), 359-371.

Tegos, S., Kioumousidou, M., Vivami, M., Kioutsiouki, D., Gkoumas, D y Tsiatsos, T. (2014). HistoryLand: A serious game for historylearning in primary Education. Proceeedings of the European Conference on Games-based Lerning. (4) 838-842.

Téllez, D. (2015). Plague inc: Pandemias, videojuegos y enseñanza-aprendizaje de las ciencias sociales. Innovaciones Didácticas. Departamento de ciencias de la Educación, Universidad de la Rioja. (14), 135-142. DOI: 10.1344 / ECCSS2015.14.1 
Villaseñor, A.Y., Sánchez, E.M., Santana, P.C. y Rodríguez, M.A. (2013). Juego serio con interacción natural para la activación física en niños de primaria. Congreso Internacional de las Ciencias de la educación. Universidad de Colima, Facultad de Ciencias de la Educación y Facultad de Pedagogía. México. 545-552.

Valderrama Ramos, J. (2012) Los videojuegos: Conectar al alumno para a aprender. Sinética Revista Electrónica de Educación, (39), 1-15.

Villacís, C. J., Fuertes, W. M., Bustamante, C. A., Zambrano, M. E., Torres, E. P., Aules, H. M. y Basurto, M. O. (2015). Optimización del juego tres en raya con niveles de dificultad utilizando heurística de inteligencia artificial. Novas Prácticas em informacao e conhecimento,Universidade Federal do Paraná 3(2), 95-106.

Yagüe, S., Yagüe, S., Lecuona, A. y Sanz, R. (2016) Los videojuegos en el tratamiento fisioterapéutico de la parálisis cerebral. Asociación Española de Fisioterapeutas. España. (38) 6. 295-302. DOI:

10.1016/j.ft.2015.11.005

Zhao, Z., y Linaza, J. L. (2015). Relevance of videogames in the learning and developmen of young children. Electronic Journal of Research in Educational Psichology(36), 301-318.

Esta obra está bajo una Licencia Creative Commons Attribución-NoCommercial 4.0 International

(cc) BY-NC 